\title{
TERT promoter mutations and their correlation with BRAF and RAS mutations in a consecutive cohort of 145 thyroid cancer cases
}

\author{
ANDREA CACCIATO INSILLA ${ }^{1}$, AGNESE PROIETTI $^{1}$, NICLA BORRELLI ${ }^{1}$, \\ ELISABETTA MACEROLA ${ }^{1}$, CRISTINA NICCOLI $^{1}$, PAOLO VITTI $^{2}$, PAOLO MICCOLI $^{3}$ and FULVIO BASOLO ${ }^{1}$
}

Departments of ${ }^{1}$ Surgical Pathology, ${ }^{2}$ Endocrinology and ${ }^{3}$ Endocrine Surgery, University Hospital of Pisa, I-56126 Pisa, Italy

Received October 3, 2016; Accepted February 13, 2017

DOI: 10.3892/ol.2017.7675

\begin{abstract}
Papillary thyroid carcinoma (PTC) is the most common type of endocrine malignancy and accounts for $\sim 80 \%$ of thyroid carcinomas in adults and $90 \%$ in children. Risk stratification is important for identifying patients at higher risk and, for this reason, recent advances in molecular genetics of thyroid cancer can be applied to provide novel biomarkers useful in understanding tumor behavior. B-Raf proto-oncogene, serine/threonine kinase (BRAF) and rat sarcoma (RAS) mutations have been widely studied and appear to have an important role in thyroid tumorigenesis. Somatic telomerase reverse transcriptase (TERT) promoter mutations have been recently identified in several types of malignant tumors, including thyroid neoplasia; however, the actual role of TERT mutations in thyroid tumorigenesis is still under debate. In the present study, the mutational status of BRAF, RAS and TERT was analyzed in order to elucidate the roles of these genes in thyroid tumorigenesis. The TERT mutational analysis was also correlated with an immunohistochemical study of TERT protein expression. According to the literature, our data provide evidence of the BRAF and RAS roles in thyroid tumorigenesis, supporting an association between BRAF (V600E) mutations and the more aggressive clinical and pathological features of thyroid tumors. By contrast, TERT mutations were not significantly associated with any clinical parameters; therefore, its role in initial tumorigenesis should be further investigated.
\end{abstract}

\section{Introduction}

Papillary thyroid carcinoma (PTC) is the most common type of endocrine malignancy (1). PTC accounts for $\sim 80 \%$ of thyroid

Correspondence to: Professor Fulvio Basolo, Department of Surgical Pathology, University Hospital of Pisa, Via Roma 57, I-56126 Pisa, Italy

E-mail: fulvio.basolo@med.unipi.it

Key words: telomerase reverse transcriptase promoter, B-Raf proto-oncogene, serine/threonine kinase, rat sarcoma, molecular biology, thyroid carcinomas in adults and $90 \%$ in children; patients are usually 20-50 years old, but individuals of any age may be affected (1). PTC is more common in females, with a prevalence 2.9-times higher in women than in men (2). The incidence of this human tumor type has been grown rapidly over the past three decades with a 2.3 -fold increase in the total number of patients, a trend that has been observed in numerous countries across Europe, Asia, Oceania, North and South America (3). Well differentiated papillary thyroid carcinomas (WDPTC) are associated with superior survival statistics when treated appropriately with surgery, radioiodine ablation and thyroid suppression therapy; however, the occurrence of aggressive WDPTC is not a rare event (4) and there is an increasing requirement for more accurate prognostic tools to predict the possible disease outcome. Risk stratification is important for identifying patients at a higher risk of recurrence or distant metastasis so more aggressive therapy and monitoring can be implemented.

In addition to the known clinical and histopathological risk factors, recent advances in the molecular genetics of thyroid cancer may be applied to identify certain novel biomarkers useful for understanding the tumor behavior. B-Raf proto-oncogene, serine/threonine kinase (BRAF) mutations are known to have an important role in PTC tumorigenesis and appear to be correlated with the outcome of the disease (5-7). $\mathrm{BRAF}$ is a serine/threonine protein kinase, encoded on chromosome 7q34, which activates the mitogen activated protein kinase (MAPK)/extracellular signal-related kinase-signaling pathway, which affects cell division, proliferation and differentiation. The BRAF mutation exists in PTCs and PTC-derived anaplastic thyroid cancer (ATCs), but it has not been described in follicular (FTC) or medullary (MTC) thyroid carcinoma, or in normal thyroid tissue (6). Occasionally, a few uncommon BRAF mutations have been reported in benign thyroid neoplasia $(8,9)$. The BRAF mutation has been identified in $29-69 \%$ of PTCs and in $>80 \%$ of PTCs of the tall cell variant, and the correlation between the BRAF mutation and the poorer clinicopathological characteristics of PTC has been demonstrated (5,7). Approximately $90 \%$ of BRAF somatic mutations consist of a T to A substitution at codon 600 (p.V600E) (10). Rat sarcoma (RAS) mutations have also been studied; RAS mutations are identified with variable frequency in all types of thyroid follicular cell-derived tumors (11). They occur in $10-20 \%$ of PTCs, especially in the follicular variant, in $40-50 \%$ of conventional-type follicular carcinomas and $20-40 \%$ of 
conventional-type follicular adenomas (6). In general, previous studies have demonstrated that the presence of RAS mutations in a thyroid nodule provides evidence for neoplasia; however, it does not establish a diagnosis of malignancy $(6,11)$.

Beyond BRAF and RAS, additional studies regarding non-coding mutations in cancer have underlined the importance of the human telomerase reverse transcriptase (hTERT) promoter mutation $(12,13)$. Telomerase is a ribonucleoprotein polymerase that maintains the telomere repeat TTAGGG at the ends of chromosomes, and consists of a protein with reverse transcriptase activity, TERT, and an RNA component that serves as a template (13). Initially identified in melanoma, TERT promoter mutations appear to have a vital role in the pathogenesis of other types of neoplasm, including bladder cancer, hepatocellular carcinoma, liposarcoma, central nervous system tumors and thyroid cancer [WDPTC or poorly differentiated (PDTC) and ATC] (14). Recent studies have revealed an association between the TERT mutation and certain clinical features of patients, including an older age at diagnosis, male sex and tumor size, particularly in WDPTCs (13). In addition, the coexistence of TERT and BRAF/RAS mutations has been taken into consideration and certain authors emphasized how TERT mutations appear to be more frequent in BRAF-mutated WDPTC, in particular in those with a V600E mutation, as compared with in BRAF-wild type (WT) carcinoma (13). It is not currently clear whether this association has a clinical impact: Liu et al (15) revealed that TERT mutations in thyroid cancer are particularly prevalent in BRAF-V600E mutated PTC, having a role in the de-differentiation, progression and aggressiveness of the tumor (14). Conversely, additional studies identified no significant differences in the outcome among cancer cases with TERT/BRAF mutations, and those cases with only TERT mutations (13).

The present study focused on the expression of TERT in a large series of thyroid cancer cases, investigating a possible correlation between its expression and certain features of the tumor. In addition, the expression of TERT according to BRAF and RAS mutations was evaluated.

\section{Materials and methods}

Patients and tumor specimens. A large series of consecutive primary papillary thyroid tumors were obtained from 145 patients (106 females and 39 males) who underwent surgical resection at the University Hospital of Pisa (Pisa, Italy) between January and December 2010. Formalin fixed and paraffin-embedded thyroid specimens from all the 145 patients were sliced with a microtome, and routine hematoxylin and eosin staining was performed. They were stored in archives and were retrospectively reviewed. All 145 cases were WDPTCs; in particular, 79 were follicular variants (FVPTC), 47 were classic variants (CVPTC), 15 were tall cell variants (TCPTC), two were solid/trabecular variants, one was a macrofollicular variant and one was a Hurthle cell variant. The age of the patients ranged between 10 and 78 , with a mean age of 46. All the specimens were reviewed by two pathologists (A.C.I. and A.P.) to confirm the diagnosis. This study was approved by the institutional review board, and informed consent was obtained from all patients.
DNA purification. Serial $10 \mu \mathrm{m}$-thick tissue sections were obtained from paraffin blocks for DNA extraction from the primary tumor tissue. Hematoxylin and eosin reference slides were marked to identify the area of interest with neoplastic cells; afterwards neoplastic areas were macro-dissected. For the extraction of the DNA, embedded sections were deparaffinized in xylene, dehydrated through a graded series of alcohols, and processed using a diaminobenzidine detection system (Ventana Medical Systems, Inc., Tucson, AZ, USA), following the manufacturer's protocol. DNA was purified using the Qiagen RNeasy FFPE kit (Qiagen GmbH, Hilden, Germany) as described by the manufacturer. DNA concentration and quality were assessed by a spectrophotometer (NanoDrop ND-1000, NanoDrop Technologies; Thermo Fisher Scientific, Inc., Waltham, MA, USA); the instrument measures absorbance at $260 \mathrm{~nm}$ (A260) to quantify DNA in samples, at $280 \mathrm{~nm}$ (A280) to verify protein contamination and at $230 \mathrm{~nm}$ (A230) for determining contamination by phenol. Ratio between A260/A280 and A260/A230 are parameters to evaluate DNA purity (A260/A280 should be $>1.7$; A260/A280 should be >1.8).

TERT, BRAF and RAS gene family mutational analysis. The DNA was evaluated for the TERT, BRAF and RAS gene family. The two more frequently identified variations of the promoter of the TERT gene (located on chromosome 5) at positions 1295228 and 1295250 are known as C228T and C250T, respectively. These mutations are located at -124 and -146-bp upstream of the ATG start codon $(13,16)$. Additionally, two low-frequency $\mathrm{CC}>\mathrm{TT}$ tandem variations have been described at positions $1295228 / 1295229(-124 /-125$ from the ATG site) and 1295242/1295243 (-138/-139 from the ATG site), which were named C228T/C229T and C242T/C243T, respectively $(13,16)$. Consequently, the TERT promoter target region was amplified via PCR using the following primer pair: TERT promoter forward 5'-CAGCGCTGCCTGAAA CTC-3'; reverse 5'-GTCCTGCCCCTTCACCTT-3' $(1,2)$. Consequently, the TERT promoter target region was amplified via PCR using the following primer pair: TERT promoter forward 5'CAGCGCTGCCTGAAACTC 3'; reverse 5' GTCCTGCCCCTTCACCTT 3' and the following reagents mixture: $10 \mathrm{mM}$ Tris- $\mathrm{HCl}, 50 \mathrm{mM} \mathrm{KCl}, 1.5 \mathrm{mM} \mathrm{MgCl}{ }_{2}$ (pH 8.3), $0.2 \mathrm{mM}$ dNTPs, 8 pmol primers and $1.25 \mathrm{U}$ AmpliTaq Gold DNA Polymerase (Thermo Fisher Scientific, Inc.) in a final volume of $20 \mu \mathrm{l}$. A total of $5 \mu \mathrm{l}$ DNA were added, and reaction was performed on a thermal cycler (SensoQuest, Gottingen, Germany) with the following protocol: Hold at $95^{\circ} \mathrm{C}$ for $5 \mathrm{~min} 50$ cycles $95^{\circ} \mathrm{C}$ for $30 \mathrm{sec}, 55^{\circ} \mathrm{C}$ for $30 \mathrm{sec}$ and $72^{\circ} \mathrm{C}$ for $45 \mathrm{sec}$. A total of $2 \mu \mathrm{l} \mathrm{PCR}$ products were used as template for reaction sequencing containing labeled nucleotide terminators $1 \mu \mathrm{l}$, sequencing buffer $2 \mu \mathrm{l}, 1 \mu \mathrm{M}$ primer $3 \mu \mathrm{l}$ and water in a final volume of $20 \mu \mathrm{l}$ (BigDye Terminator Cycle Sequencing kit; Applied Biosystem, Foster City, CA, USA). The reaction conditions were the following: Hold at $96^{\circ} \mathrm{C}$ for $10 \mathrm{~min}, 24 \mathrm{cycles}$ at $96^{\circ} \mathrm{C}$ for $10 \mathrm{sec}, 50^{\circ} \mathrm{C}$ for $5 \mathrm{sec}$, and $60^{\circ} \mathrm{C}$ for $4 \mathrm{~min}$. Then, the reaction was run for direct sequencing on a AbiPrism 3130 Genetic Analyzer (Applied Biosystem, Foster City, CA, USA). BRAF and RAS genes (NRAS, HRAS, KRAS) were analyzed by high-resolution melt analysis (HRMA) followed by direct sequencing. Approximately $80 \mathrm{ng}$ DNA 
was amplified in a final volume of $25 \mu 1$ containing $12.5 \mu 1$ Master Mix (Qiagen GmbH, Hilden, Germany) $0.8 \mu \mathrm{mol} / 1$ of each primer and $1 \mu \mathrm{l}$ EvaGreen 20X. PCR and HRMA were performed on a Rotorgene $6000^{\mathrm{TM}}$ Real Time Analyzer (Qiagen $\mathrm{GmbH}$ ). Post-amplification fluorescence melting curve analysis was performed by gradual heating of samples at a rate of $1^{\circ} \mathrm{C} / \mathrm{sec}$ from $45-95^{\circ} \mathrm{C}$. A HRMA was immediately performed from $75-85^{\circ} \mathrm{C}$ rising at $0.1^{\circ} \mathrm{C} / \mathrm{sec}$. The resulting data were analyzed using Rotor-Gene Series software version 1.7 (Qiagen $\mathrm{GmbH}$ ). PCR products of all samples with altered melting curves, together with a number of non-altered ones, were sequenced as described for TERT promoter analysis.

Immunohistochemical studies. Samples with the best tumor/normal tissue ratio and/or samples without factors that may invalidate immunohistochemistry (necrosis, calcifications, fibrosis) from the paraffin block as the best representation of the tumors was selected for analysis for each case. The immunohistochemical analyses were performed automatically using the Ventana Bench-mark immunostaining system (Ventana Medical Systems, Inc.). Paraffin-embedded tissue sections (3-5 $\mu \mathrm{m})$ were deparaffinized in xylene, rehydrated through a graded series of ethanol and processed using a diaminobenzidine detection system (Ventana Medical Systems, Inc.), following the manufacturer's instructions. TERT immunostaining was performed using a rabbit polyclonal antibody produced by repeated immunizations with a synthetic peptide corresponding to a region near the carboxy-terminal end of hTERT (no. AF018167; Rockland Immunochemicals, Inc., Limerick, PA, USA) at a dilution of 1:300. In order to test the specificity and sensitivity of the antibody, control neoplastic tissues (astrocytoma) from the archives were also stained. The immunostaining of all the samples was evaluated independently by two surgical pathologists; each pathologist assessed the intensity and extent of immunoreactivity for each case. Disparate scores between the two investigators were observed in $<10 \%$ of the samples and a consensus was achieved in all cases following discussion. In case of disagreement the slides were evaluated again with a multi-ocular microscope. All the samples were scored based on the percentage and intensity of the positive neoplastic cells. The extension of the positive reaction was classified into three grades (1, 2 or 3 ) as follows: Grade 1, a positive reaction was detected in $<20 \%$ of the neoplasm; grade 2 , a positive reaction between $21-50 \%$; grade 3 , a positive reaction was detected in $>50 \%$ of the neoplasm. The staining intensity was also classified in three grades, as follows: Grade 1, weak intensity; grade 2, moderate intensity; grade 3 , strong intensity (Table I). Subsequently, a total score ranging from 2-6 was obtained by adding the scores of the two categories analyzed (Fig. 1).

A z-score was also calculated, taking into account the intensity of staining and the percentage of immunoreactive cells. All the cases were divided into two groups, defined as positive or negative, depending on the z-score obtained: All the cases with a $\mathrm{z}$-score $>0$ were defined as positive; all the cases with a $\mathrm{z}$-score $<0$ were defined as negative.

Statistical analysis. Statistical analysis was performed using IBM SPSS Statistical software (version 17.0.1; IBM SPSS,
Table I. Classification of IHC grade and stain intensity.

\begin{tabular}{lcl}
\hline Grade & Positive area extension, $\%$ & Intensity \\
\hline 1 & $<20$ & Weak \\
2 & $21-50$ & Moderate \\
3 & $>50$ & Strong \\
\hline
\end{tabular}

IHC, immunohistochemistry.

Armonk, NY, USA). A Shapiro-Wilk test was performed to verify the normality of distributions. Correlations between clinical features were evaluated using Fisher's exact test, $\chi^{2}$ test or a two-tailed t-test, as appropriate. $\mathrm{P}<0.05$ was considered to indicate a statistically significant difference.

\section{Results}

Clinical-pathological features of 145 cases of PTC. Several clinical features were analyzed for all tumors. A total of 76 cases were capsulated tumors and 64 out of these 76 capsulated tumors resulted FVPTC. Among the other 69 non-capsulated cases, 36 exhibited infiltration of the perithyroidal soft tissues; in particular, 21 were CVPTCs, 11 were TCPTCs and 4 were FVPTCs. A total of 16/145 cases had lymph node metastasis at the time of diagnosis (12 CVPTCs, 3 TCPTCs and 1 FVPTC). Finally, 7/145 cases had embolization at the diagnosis, of which 6 were CVPTCs and 1 was TCPTC.

$B R A F$ and RAS mutation. Among all 145 tumors analyzed, a BRAF mutation was detected in 53 cases, corresponding to $36.6 \%$ of the entire cohort; in particular, 31 BRAF mutated tumors were CVPTCs, 12 were TCPTCs and 10 were FVPTCs (Table II). All the CVPTCs, as well as all the TCPTCs, carried a V600E mutation; instead, among the 10 BRAF-mutated FVPTCs, 6 had a V600E mutation, 2 a K601E mutation, 1 presented with a deletion (V600_K601delinsE) and 1 with a T599I mutation. Comparing the presence of BRAF mutation with clinical-pathological features we observed 27 cases with involvement of the perithyroidal tissues, 6 cases with embolization and 11 with lymph nodes metastasis. RAS mutation was detected in 23 cases, corresponding to $15.9 \%$ of the entire cohort and all were FVPTCs. In particular, an NRAS mutation occurred in 18/23 cases, while the remaining 5 cases were HRAS mutated (Table II). All 23 RAS mutated tumors were limited to the parenchyma without involving the periglandular soft tissues and, in particular, 22/23 presented as capsulated nodules. None of the tumors with embolization or lymph node metastasis were revealed to be RAS mutated.

TERT promoter mutation. In our study, TERT mutations were identified in 9/145 (5 CVPTCs, 2 FVPTCs and 1 TCPTC) corresponding to the $6.2 \%$ of the overall cohort. 'C228T' mutation was the most frequent alteration observed, present in 8/9 cases; the remaining one exhibited a 'C250T' mutation. All the 145 patients were divided according to the TERT mutation in 'mutated'/'non-mutated' and several clinical features were studied, the most important reported 

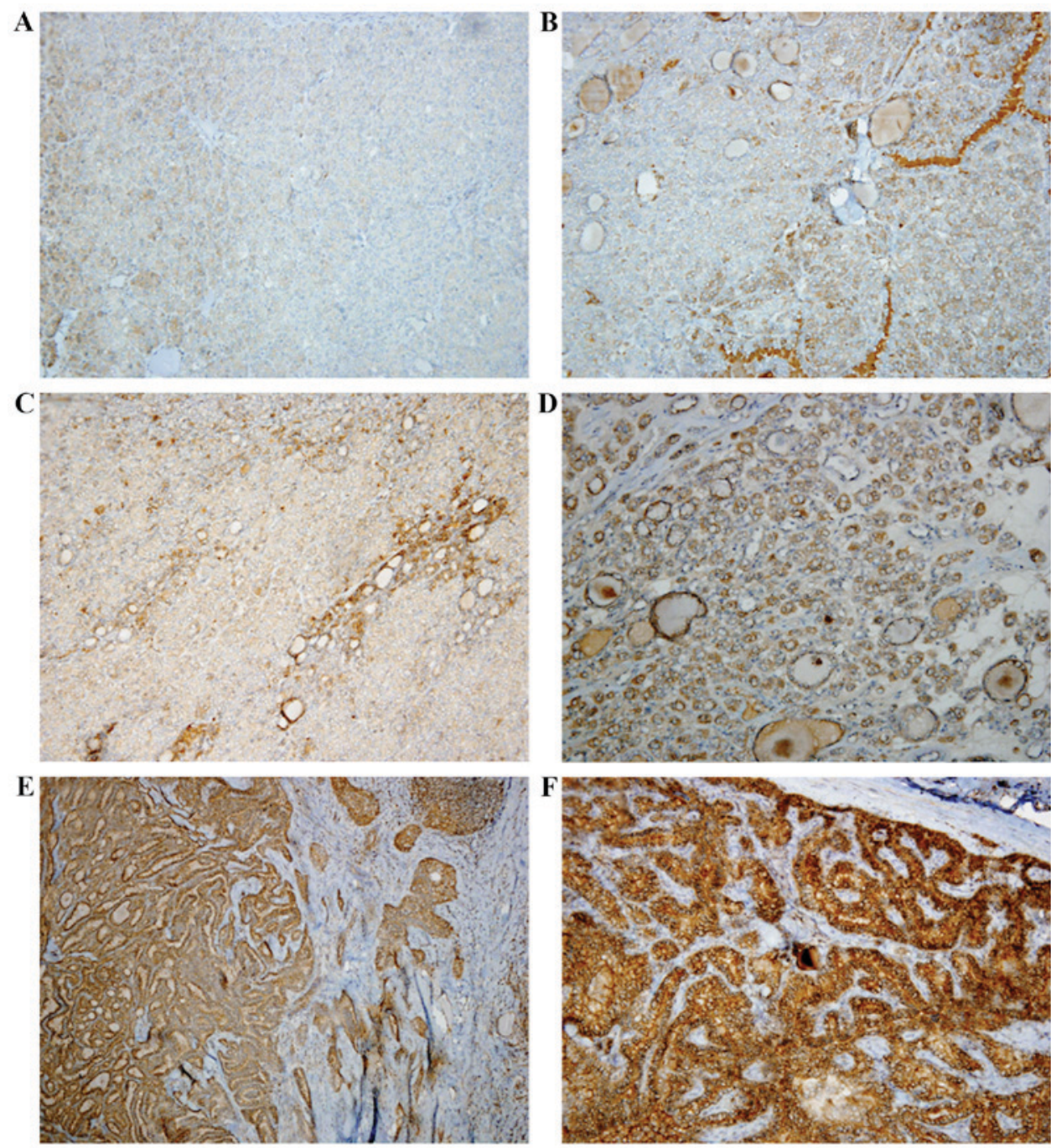

Figure 1. Immunostaining of TERT in tissue samples obtained from patients with primary papillary thyroid tumor. Scoring of TERT expression was performed as follows: (A) expression score 2 (x4); (B) expression score 3 (x10); (C) expression score 4 (x10); (D) expression score 4 (x20); (E) expression score 5 (x10); (F) expression score 6 (x20). TERT, telomerase reverse transcriptase.

in Table III. We identified a significant association with sex (66.7\% males and 33.3\% females). All the other parameters analyzed did not exhibit any association with TERT mutation.

Association of TERT promoter mutation with BRAF and RAS mutation. We analyzed the possible correlation of TERT promoter mutation with BRAF and RAS mutation. TERT mutations were identified in 5/53 BRAF mutated PTCs (9.4\%, 4 CVPTCs and 1 TCPTC): In these co-mutated cases, all the 5 BRAF mutations were V600E, while regarding TERT mutations, 4 were C228T and 1 was C250T. The other 4 TERT mutated cases were identified among the remaining 92 BRAF-WT tumors (4.4\%) and all of them exhibited a C228T mutation (Table IV). All five PTCs with BRAF and TERT mutation revealed features of a more aggressive behavior: None of them were capsulated, $4 / 5$ were infiltrating the thyroidal capsule and 3/5 the extra-thyroidal soft tissues, while $2 / 5$ had lymph node metastasis (Table V). Only one case presented with RAS and TERT mutations; it was an encapsulated, well-differentiated FVPTC with no lymph node metastasis or vascular invasion (Table VI).
TERT Immunohistochemical expression. The expression of TERT protein was evaluated in neoplastic and in normal peritumoral tissues. All the neoplastic tissue samples analyzed exhibited a cytoplasmic pattern of immunoreactivity; normal tissues had the same cytoplasmic positivity, but 2 cases also had nuclear positivity. In tumors, the mean cytoplasmic intensity of staining was 1.7 ; the mean percentage of immunoreactive cells was 1.7 as well, in accordance to similar data reported in literature (13). No significant differences in TERT protein expression were observed between mutated and WT tumors (Table VII). According to the Z-score calculated, 69 cases out of 145 were considered positive; 36 cases out of 69 had a total score (intensity plus extension) of 5 or 6: 19 were FVPTCs, 11 CVPTCs, 5 TCPTCs and 1 was a macrofollicular carcinoma. The TERT protein expression evaluated in these cases did not appear to correlate significantly with any clinical pathological aspect. Among these 36 positive cases, 16 were BRAF mutated (9 CVPTCs, 4 TCPTCs and 3 FVPTCs) while 4 were RAS mutated. Only one case resulted to be TERT mutated; this case, which presented with BRAF and TERT mutation, was a CVPTC, limited to the thyroid and with neither the presence of embolization nor lymph node metastasis. 
Table II. Clinicopathological features of 145 cases of PTC.

\begin{tabular}{|c|c|c|c|c|}
\hline $\begin{array}{l}\text { Clinicopathological } \\
\text { characteristic }\end{array}$ & $\begin{array}{c}\text { CVPTC } \\
(47 / 145-32.4 \%)(\%)\end{array}$ & $\begin{array}{c}\text { FVPTC } \\
(79 / 145-54.5 \%)(\%)\end{array}$ & $\begin{array}{c}\text { TCPTC } \\
(15 / 145-10.3 \%)(\%)\end{array}$ & $\begin{array}{c}\text { OTHERS }^{\mathrm{a}} \\
(4 / 145-2.8 \%)(\%)\end{array}$ \\
\hline \multicolumn{5}{|l|}{ Sex } \\
\hline Female $(106 / 145-73.1 \%)$ & 29/47 (61.7) & $61 / 79(77.2)$ & $13 / 15(86.7)$ & $3 / 4(75)$ \\
\hline Male $(39 / 145-26.9 \%)$ & $18 / 47(38.3)$ & $18 / 79(22.8)$ & $2 / 15(13.3)$ & $1 / 4(25)$ \\
\hline \multicolumn{5}{|l|}{ Size } \\
\hline$\leq 1 \mathrm{~cm}(44 / 145-30.3 \%)$ & $17 / 47(36.2)$ & $24 / 79(30.4)$ & $3 / 15(20)$ & - \\
\hline$>1 \mathrm{~cm}(101 / 145-69.7 \%)$ & $30 / 47(63.8)$ & $55 / 79(69.6)$ & $12 / 15(80)$ & $4 / 4(100)$ \\
\hline \multicolumn{5}{|l|}{ Capsule } \\
\hline Capsulated (76/145-52.4\%) & 8/47 (17.1) & $64 / 79(81)$ & - & $4 / 4(100)$ \\
\hline Not Capsulated (69/145-47.6\%) & 39/47 (82.9) & $15 / 79(19)$ & $15 / 15(100)$ & - \\
\hline \multicolumn{5}{|l|}{ Tumor capsule invasion } \\
\hline Yes $(8 / 76-10.5 \%)$ & $1 / 47(2.1)$ & $7 / 79(8.9)$ & - & - \\
\hline No $(68 / 76-89.5 \%)$ & 46/47 (97.9) & $72 / 79(91.1)$ & $15 / 15(100)$ & 4/4 (100) \\
\hline \multicolumn{5}{|l|}{ Thyroid capsule invasion } \\
\hline Yes $(17 / 69-24.6 \%)$ & 9/47 (19.2) & $6 / 79(7.6)$ & 2/15 (13.4) & - \\
\hline No $(52 / 69-75.4 \%)$ & $38 / 47(80.8)$ & $73 / 79(92.4)$ & $13 / 15(86.6)$ & $4 / 4(100)$ \\
\hline \multicolumn{5}{|l|}{ Perithyroid soft tissue invasion } \\
\hline Yes $(36 / 69-52.2 \%)$ & $21 / 47(44.7)$ & 4/79 (5.1) & $11 / 15(73.4)$ & - \\
\hline No $(33 / 69-47.8 \%)$ & $26 / 47(55.3)$ & 75/79 (94.9) & 4/15 (26.6) & 4/4 (100) \\
\hline \multicolumn{5}{|l|}{ Embolization } \\
\hline Yes $(7 / 145-4.8 \%)$ & $6 / 47(12.8)$ & - & $1 / 15(6.7)$ & - \\
\hline No $(138 / 145-95.2 \%)$ & $41 / 47(87.2)$ & $79 / 79(100)$ & $14 / 15(93.3)$ & $4 / 4(100)$ \\
\hline \multicolumn{5}{|l|}{ Lymph node metastasis } \\
\hline Yes $(16 / 145-11 \%)$ & $12 / 47(25.5)$ & $1 / 79(1.3)$ & $3 / 15(20)$ & - \\
\hline No $(129 / 145-89 \%)$ & $35 / 47(74.5)$ & 78/79 (98.7) & $12 / 15(80)$ & $4 / 4(100)$ \\
\hline \multicolumn{5}{|l|}{ BRAF } \\
\hline Mutated (53/145-36.6\%) & $31 / 47(66)$ & $10 / 79(12.7)$ & $12 / 15(80)$ & - \\
\hline WT (92/145-63.4\%) & $16 / 47(34)$ & $69 / 79(87.3)$ & $3 / 15(20)$ & 4/4 (100) \\
\hline \multicolumn{5}{|l|}{ RAS } \\
\hline Mutated (23/145-15.9\%) & - & 23/79 (29.1) & - & - \\
\hline WT (122/145-84.1\%) & $47 / 47(100)$ & $56 / 79(70.9 \%)$ & $15 / 15(100)$ & 4/4 (100) \\
\hline
\end{tabular}

aOthers: 1 Macrofollicular variant, 1 Hurtle cell variant, 2 Solid and Trabecular variant. BRAF, B-Raf proto-oncogene, serine/threonine kinase; RAS, rat sarcoma; WT, wild type; PTC, papillary thyroid carcinoma, FV, follicular variant; CV; classic variant; TC, tall cell variant.

\section{Discussion}

Thyroid tumor is the most common endocrine malignant cancer, with an increasing incidence all around the world. WDPTCs are usually indolent lesions with low metastatic potential and a 5-year survival rate of approximately $98 \%$ (17), but a small significant percentage behave aggressively, developing metastases and possibly leading the patient to death (18). Numerous studies have been carried on to identify prognostic markers able to discriminate aggressive PTCs from those with a more indolent outcome, to identify patients at higher risk and to better define the therapeutic approaches, avoiding overtreatments $(5,19)$. BRAF mutation is already known to have an important role in PTC tumorigenesis and prognosis, in particular with his variant V600E that occurs in almost the
$45 \%$ of patients. The presence of this latter mutation appears to correlate with a higher risk of recurrence and probably with a more aggressive behavior of the tumor, while a association between BRAF mutation status and PTC-related mortality is still under debate $(5,17)$. According to the literature, in our study we observed the presence of a BRAF mutation in the $36.6 \%$ of cases and, among all the BRAF-mutated tumors, the $94.3 \%$ had a V600E mutation. At the histological examination, the majority of these V600E-mutated cases (83\%) were CVPTCs or TCPTCs and all these tumors exhibited a tendency to behave in a more aggressive way. In particular, several tumors with evidence of extrathyroidal extension $(27 / 36,75 \%)$ were V600E mutated as well as numerous cases with lymph nodes metastasis $(11 / 15,73.4 \%)$ or embolization (6 in $7,85.7 \%$ ). Among all the BRAF mutated cases, four presented with a rare 
Table III. Clinicopathological features of the 145 TERT mutated and WT PTC.

\begin{tabular}{lcc}
\hline $\begin{array}{l}\text { Clinicopathological } \\
\text { characteristic }\end{array}$ & TERT $^{(\mathrm{MUT})} /$ TERT $^{(\mathrm{WT})}$ & P-value \\
\hline Sex & & $<0,05$ \\
Male & $6 / 33$ & \\
Female & $3 / 103$ & \\
Cap & $3 / 73$ & $\mathrm{NS}$ \\
TCI & $1 / 16$ & $\mathrm{NS}$ \\
PSTI & $3 / 33$ & $\mathrm{NS}$ \\
Lymph & $2 / 14$ & $\mathrm{NS}$ \\
\hline
\end{tabular}

Cap, capsule invasion; TCI, thyroidal capsule invasion; PSTI, perithyroidal soft tissue invasion; Lymph, lymph node metastasis; Mut, mutated; TERT, telomerase reverse transcriptase; PTC, papillary thyroid carcinoma; WT, wild type; NS, not significant.

Table IV. Correlation between TERT and BRAF mutations.

\begin{tabular}{lccc}
\hline & \multicolumn{2}{c}{ TERT Mutation } & \\
\cline { 2 - 3 } BRAF status & Present (9) (\%) & Absent (136) (\%) & P-value \\
\hline BRAF & & & NS \\
Mutated (53) & $5(55.6)$ & $48(35.3)$ & \\
WT (92) & $4(44.4)$ & $88(64.7)$ & \\
& &
\end{tabular}

TERT, telomerase reverse transcriptase; BRAF, B-Raf proto-oncogene, serine/threonine kinase; WT, wild type; NS, not significant.

Table V. Clinicopathological features of the five BRAF and TERT mutated PTCs.

\begin{tabular}{lcccc}
\hline BRAF/TERT ratio & Cap & TCI & PSTI & Lymph \\
\hline BRAF $^{(\mathrm{MUT})} / \mathrm{TERT}^{(\mathrm{MUT})}$ & $0 / 5$ & $4 / 5$ & $3 / 5$ & $2 / 5$ \\
\hline
\end{tabular}

Cap, capsule invasion; TCI, thyroidal capsule invasion; PSTI, perithyroidal soft tissue invasion; Lymph, lymph node metastasis; Mut, mutated; PTC, papillary thyroid carcinoma

BRAF mutation: 2 cases with a K601E mutation, 1 with the 'V600_K601delinsE' deletion and 1 with a T599I mutation. As reported in a recent study, rare BRAF mutations appear to be more frequent in thyroid tumors with a more indolent behavior, such as FVPTCs and the prevalence of aggressive clinicopathological features is lower in these tumors than in V600E mutated ones. According to these data, all our PTCs with a rare BRAF mutation were encapsulated FVPTC and none of them exhibited signs of clinical aggressiveness like extrathyroidal extension, vascular invasion or lymph node metastasis (20). The role of RAS oncogene in thyroid tumorigenesis has been established, as well: RAS mutations occur up
Table VI. Correlation between TERT and RAS mutations.

\section{TERT Mutation}

\begin{tabular}{lccc} 
Phenotype & Present (9) (\%) & Absent (136) (\%) & P-value \\
\hline RAS & & & NS \\
Mutated (23) & $1(11.2)$ & $19(14)$ & \\
WT (122) & $8(88.8)$ & $117(86)$ &
\end{tabular}

TERT, telomerase reverse transcriptase; RAS, rat sarcoma; WT, wild type; NS, not significant.

Table VII. Correlation between TERT IHC expression, BRAF and RAS mutations.

\begin{tabular}{llrr}
\hline & \multicolumn{2}{c}{ TERT IHC Expression } & \\
\cline { 2 - 3 } & $\begin{array}{l}\text { Positive } \\
(>0 ; 69)\end{array}$ & $\begin{array}{r}\text { Negative } \\
(<0 ; 76)\end{array}$ & P-value \\
Phenotype & & & NS \\
\hline BRAF & $28(40.6)$ & $25(32.9)$ & \\
Mutated (53) & $41(59.4)$ & $51(67.1)$ & \\
WT (92) & & & NS \\
RAS & $11(15.9)$ & $9(11.8)$ & \\
Mutated (20) & $58(84.1)$ & $67(88.2)$ & \\
WT (125) & & & \\
\hline
\end{tabular}

Positive, z-score $>0$; Negative: $z$-score $<0$. TERT, telomerase reverse transcriptase; IHC, immunohistochemistry; BRAF, B-Raf proto-oncogene, serine/threonine kinase; RAS, rat sarcoma; WT, wild type; NS, not significant.

to $45 \%$ of follicular thyroid cancer cases as well as in $30-40 \%$ of follicular variant papillary thyroid carcinoma; in addition, they have also been described in anaplastic thyroid cancer cases and, occasionally, in benign adenomas $(6,11)$. Several studies have demonstrated that RAS mutated WDPTCs, without other coexisting genetic alterations, generally lack aggressive behavior $(6,11)$. In our study, all the 23 RAS-mutated neoplasms were a follicular variant of papillary thyroid carcinoma: 22 out of 23 were encapsulated forms of cancer and only two cases exhibited a clear invasion of the neoplastic capsule; embolization or lymph node metastasis were absent in all the 23 cases analyzed. These data appear to confirm that RAS mutated cancer tends to behave in a less aggressive manner. One of the 23 RAS mutated cases also presented with a TERT (C228T) mutation but, differently from what observed in BRAF-TERT co-mutated cases, the RAS-TERT co-mutated one did not exhibit any sign of aggressiveness. Beyond BRAF and RAS, numerous researchers have started to focus on certain new genes mutations, such as the TERT promoter one. This mutation has been described in numerous different kinds of tumors, such as melanoma, bladder cancer, hepatocellular carcinoma, squamous cell carcinoma, liposarcoma, a subset of central nervous tumors and, recently, thyroid tumors $(10,14)$. The TERT promoter mutations have been reported in PTCs 
and also in FTCs, while they do not appear to have a role in the tumorigenesis of MTCs $(13,15)$. In particular, the average TERT mutation percentage for WDPTCs reported in the literature amount to $13,7 \%$, ranging from $8-25 \%$; the average percentage for PDTCs and ATCs are respectively of $34,8 \%$ and $38,4 \%$ (13). The C228T mutation is the most common one, compared with the C250T $(12,13,15)$. In our study, we analyzed a large series of 145 consecutive PTCs considering the BRAF, RAS and TERT mutation and evaluating a possible correlation of these alterations with certain clinical features. All the BRAF mutations detected were V600E, except for four FVPTCs (2 K601E, 1 deletion and 1 T599I). In addition, 23 cases out of 145 were RAS mutated, 18 N-RAS and 5 H-RAS and all of them were FVPTCs. According to the results obtained, our cohort appeared to be representative of the general population, with data in line with the literature. Nonetheless, we observed only 9 cases out of 145 with TERT mutation, corresponding to the $6.2 \%$. In these last few years, certain authors have pointed out an association between the TERT mutation and clinical aspects such as the older age at diagnosis, male sex and tumor size, especially in WDPTC. In this cohort of PTCs, TERT promoter mutations were not identified to correlate significantly with any clinical feature, except for the sex, confirming a prevalence in males. certain further studies underlined how TERT mutations appear to be more frequent in BRAF-mutated (particularly V600E) than in BRAF-WT WDPTC (13). However, it is not clear whether this association has a clinical impact: Liu et al (15) for instance, demonstrated that TERT mutations in thyroid cancer are particularly prevalent in BRAF-V600E mutated PTCs, having a role in the de-differentiation, progression and aggressiveness of the tumor (15). Instead, additional studies revealed no differences in the outcome among cancer cases with mutations (TERT/BRAF) and those with only TERT mutation (13). According to these studies, in our group the coexistence of BRAF and TERT mutations was not significantly associated with any clinical parameter, probably due to the small number of cases analyzed. However, we have noticed how all the 5 PTCs with BRAF and TERT mutations had a tendency to behave in a more aggressive way. Considering our results and all the data reported in literature, it is reasonable to think that TERT mutations are likely to have a role in de-differentiation of WDPTCs but their contribution in the initial tumorigenesis could be uncertain. Furthermore, as suggested by certain authors, the possible implication of TERT mutation in the aggressiveness of WDPTCs could concern only certain particular histotypes (15). The hypothesis that TERT mutation could correlate more with the aggressiveness than the tumorigenesis of the thyroid tumor appears also to be supported by the preferential occurrence reported of TERT promoter mutations in BRAF mutated PTCs and, over all, in PDTCs and ATCs $(15,18,21,22)$; in particular, it has been supposed how TERT promoter mutation may join the mechanism involving the MAPK signaling, supporting the action of the BRAF mutation (11). In our study, among the few TERT mutated cases reported, the $55.6 \%$ were associated with a BRAF mutation and, even if the co-mutated PTCs had different clinical features, all of them appeared to have a tendency to behave in a more aggressive way presenting the infiltration of the extra-thyroidal soft tissues or lymph nodes metastasis. However, immunohistochemical results concerning the TERT protein expression did not appear to correlate significantly neither with BRAF nor RAS mutation.

The purpose of this study was to establish a possible clinical impact of the TERT promoter mutation in thyroid cancer cases. Although a cohort of cases representative of the general population, as demonstrated by the data regarding BRAF and RAS mutation, we identified a rate of TERT mutation far less than the average reported in literature. In addition, TERT mutations lonely and the coexistence of BRAF or RAS and TERT mutations were not significantly associated with any clinical parameter, probably due to the small number of mutated cases observed. The evaluation of the TERT protein expression did not reveal any particular correlation with clinical pathological aspects, as well. However, we observed how cancer cases with TERT and BRAF mutation appeared to behave in a more aggressive way, suggesting a possible role of TERT mutation in the aggressiveness of the neoplasia. On the contrary, in our opinion, its role in the initial tumorigenesis should be more investigated.

\section{References}

1. Erickson Lori A: Papillary thyroid carcinoma. Atlas of Endocrine Pathology: 31-50, 2014.

2. Rahbari R, Zhang L and Kebebew E: Thyroid cancer gender disparity. Future Oncol 6: 1771-1779, 2010.

3. Sipos JA and Mazzaferri EL: Thyroid cancer epidemiology and prognostic variables. Clin Oncol (R Coll Radiol) 22: 395-404, 2010

4. Caron NR and Clark OH: Papillary thyroid cancer. Curr Treat Options Oncol 7: 309-319, 2006.

5. Xing M, Westra WH, Tufano RP, Cohen Y, Rosenbaum E, Rhoden KJ, Carson KA, Vasko V, Larin A, Tallini G, et al: BRAF mutation predicts a poorer clinical prognosis for papillary thyroid cancer. J Clin Endocrinol Metab 90: 6373-6379, 2005.

6. Nikiforov YE: Molecular diagnostics of thyroid tumors. Arch Pathol Lab Med 135: 569-577, 2011.

7. Pelizzo MR, Dobrinja C, Casal Ide E, Zane M, Lora O, Toniato A, Mian C, Barollo S, Izuzquiza M, Guerrini J, et al: The role of $\mathrm{BRAF}(\mathrm{V} 600 \mathrm{E})$ mutation as poor prognostic factor for the outcome of patients with intrathyroid papillary thyroid carcinoma. Biomed Pharmacother 68: 413-417, 2014.

8. Afkhami M, Karunamurthy A, Chiosea S, Nikiforova MN, Seethala R, Nikiforov YE and Coyne C: Histopathologic and clinical characterization of thyroid tumors carrying the BRAF K601E mutation. Thyroid 26: 242-247, 2016.

9. Jara SM, Bhatnagar R, Guan H, Gocke CD, Ali SZ and Tufano RP: Utility of BRAF mutation detection in fine-needle aspiration biopsy samples read as 'suspicious for papillary thyroid carcinoma'. Head Neck 37: 1788-1793, 2015.

10. Macerola E, Loggini B, Giannini R, Garavello G, Giordano M, Proietti A, Niccoli C, Basolo F and Fontanini G: Coexistence of TERT promoter and BRAF mutations in cutaneous melanoma is associated with more clinicopathological features of aggressiveness. Virchows Arch 467: 177-184, 2015.

11. Xing M: Clinical utility of RAS mutations in thyroid cancer: A blurred picture now emerging clearer. BMC Med 14: 12, 2016.

12. Vinagre J, Almeida A, Pópulo H, Batista R, Lyra J, Pinto V, Coelho R, Celestino R, Prazeres H, Lima L, et al: Frequency of TERT promoter mutations in human cancers. Nat Commun 4: 2185, 2013.

13. Muzza M, Colombo C, Rossi S, Tosi D, Cirello V, Perrino M, De Leo S, Magnani E, Pignatti E, Vigo B, et al: Telomerase in differentiated thyroid cancer: Promoter mutations, expression and localization. Mol Cell Endocrinol 399: 288-295, 2015.

14. Park CK, Lee SH, Kim JY, Kim JE, Kim TM, Lee ST, Choi SH, Park SH and Kim IH: Expression level of hTERT is regulated by somatic mutation and common single nucleotide polymorphism at promoter region in glioblastoma. Oncotarget 5: 3399-3407, 2014.

15. Liu X, Bishop J, Shan Y, Pai S, Liu D, Murugan AK, Sun H, El-Naggar AK and Xing M: Highly prevalent TERT promoter mutations in aggressive thyroid cancers. Endocr Relat Cancer 20: 603-610, 2013. 
16. Horn S, Figl A, Rachakonda PS, Fischer C, Sucker A, Gast A, Kadel S, Moll I, Nagore E, Hemminki K, et al: TERT promoter mutations in familial and sporadic melanoma. Science 339: 959-961, 2013.

17. Yarchoan M, LiVolsi VA and Brose MS: BRAF mutation and thyroid cancer recurrence. J Clin Oncol 33: 7-8, 2015.

18. Gandolfi G, Ragazzi M, Frasoldati A, Piana S, Ciarocchi A and Sancisi V: TERT promoter mutations are associated with distant metastases in papillary thyroid carcinoma. Eur J Endocrinol 172: 403-413, 2015.

19. Esserman LJ, Thompson IM Jr and Reid B: Overdiagnosis and overtreatment in cancer: An opportunity for improvement. JAMA 310: 797-798, 2013

20. Torregrossa L, Viola D, Sensi E, Giordano M, Piaggi P, Romei C, Materazzi G, Miccoli P, Elisei R and Basolo F: Papillary thyroid carcinoma with rare exon $15 \mathrm{BRAF}$ mutation has indolent behavior: A single-institution experience. J Clin Endocrinol Metab 101: 4413-4420, 2016.
21. Shi X, Liu R, Qu S, Zhu G, Bishop J, Liu X, Sun H, Shan Z, Wang E, Luo Y, et al: Association of TERT promoter mutation 1,295,228 C $>$ T with BRAF V600E mutation, older patient age and distant metastasis in anaplastic thyroid cancer. J Clin Endocrinol Metab 100: E632-E637, 2015.

22. Xing M, Liu R, Liu X, Murugan AK, Zhu G, Zeiger MA, Pai S and Bishop J: BRAF V600E and TERT promoter mutations cooperatively identify the most aggressive papillary thyroid cancer with highest recurrence. J Clin Oncol 32: 2718-2726, 2014.

(c) (i) (3) $($ This work is licensed under a Creative Commons Attribution-NonCommercial-NoDerivatives 4.0 International (CC BY-NC-ND 4.0) License. 